\title{
Territoriales Asyl, Non-Refoulement und das souveräne Recht zur Grenzkontrolle
}

\section{Einführung}

Dieser Beitrag ist den Grundlagen des völkerrechtlichen Flüchtlingsschutzes gewidmet. Im Zentrum steht das Konzept staatlicher Herrschaft, die innerhalb der eigenen Staatsgrenzen souverän und für die Zustände auf dem eigenen Staatsgebiet verantwortlich ist. Daraus folgen das Recht zur Grenzkontrolle ebenso wie das Konzept des territorialen Asyls sowie der völkerrechtliche Mindestschutz in Form eines Ab- und Rückschiebungsverbots, das sogenannte Non-Refoulement. Im Vordergrund stehen das geltende Völkerrecht und die ihm zugrunde liegenden Konzepte; deren Entwicklung wird aber immer wieder historisch eingeordnet.

\section{Der souveräne Territorialstaat}

Das geltende Völkerrecht wird noch immer vom Konzept des souveränen Territorialstaats geprägt. Dieses Konzept gehört der klassischen Epoche des Völkerrechts an, die mit dem Westfälischen Frieden begann und um die Wende vom 19. zum 20. Jahrhundert ihren Höhepunkt erreichte. Das klassische Völkerrecht geht von sesshaften Personenverbänden aus, in denen Herrschaft nicht mehr allein personenbezogen ist, sondern sich insbesondere auch auf ein bestimmtes Gebiet bezieht. Dies spiegelt sich in der Drei-Elemente-Lehre wider, die den heutigen völkerrechtlichen Staatsbegriff bestimmt. Ein Staat charakterisiert sich danach durch ein Staatsgebiet, ein Staatsvolk und eine unabgeleitete Staatsgewalt, die in dem Sinne souverän ist, dass sie nur dem Völkerrecht unterworfen ist. ${ }^{1}$

In Deutschland wird die Drei-Elemente-Lehre regelmäßig auf den Staatsrechtler Georg Jellinek zurückgeführt, der den Staat in seiner 1900 erstmals erschienenen „Allgemeinen Staatslehre“ zusammenfassend als „die mit ursprünglicher Herrschermacht ausgestattete Ge-

${ }^{1}$ Vgl. Andreas von Arnauld, Völkerrecht, Heidelberg ${ }^{32016, ~ R n . ~} 72$ und Rn. 89; Jan Klabbers, International Law, Cambridge 2013, S. 69 ff. 
bietskörperschaft“ bezeichnet hat. ${ }^{2}$ Als Körperschaft setzt sich der Staat aus den Mitgliedern des Staatsvolkes zusammen, wobei er sich als Gebietskörperschaft auch über das zugehörige Staatsgebiet definiert. Schließlich zeichnet sich der Staat durch eine Herrschaft über Volk und Gebiet aus, wobei die „Herrschermacht“ ursprünglich, also souverän sein muss.

Im klassischen Völkerrecht verstand man Souveränität vor allem als Freiheit zur Herrschaft über das eigene Staatsgebiet. Völkerrechtliche Bindungen gab es für das Handeln auf eigenem Staatsgebiet bis zum Zweiten Weltkrieg kaum. Dies hat sich mit der Entwicklung des völkerrechtlichen Menschenrechtsschutzes nach 1945 verändert. ${ }^{3}$ Völkerrechtliche Menschenrechte nehmen den Staat gerade auch beim Umgang mit eigenen Staatsangehörigen auf dem eigenen Staatsgebiet in die Pflicht. Zudem wird den Menschenrechten seit dem Ende der 1970er Jahre zunehmend eine Schutzpflichtdimension entnommen mit der Folge, dass der Staat Personen, die sich auf seinem Staatsgebiet befinden, auch vor solchen Gefahren schützen muss, die von Dritten ausgehen. ${ }^{4}$ Die souveräne Herrschaft über ein Gebiet ist damit nicht nur Freiheit, sondern auch Verantwortung gegenüber den Menschen, die sich auf dem Staatsgebiet befinden.

Diesen Verantwortungsaspekt hat die International Commission on Intervention and State Sovereignty (ICISS) 2001 bekräftigt. Die ICISS war von der kanadischen Regierung nach der NATO-Intervention zugunsten des Kosovo eingesetzt worden, um die rechtlichen Implikationen einer sogenannten humanitären Intervention grundlegend aufzuarbeiten. Die Kommission ging von dem Grundgedanken aus, dass Souveränität nicht nur die Befugnis zur Herrschaft bedeute, sondern mit Verantwortung einhergehe. ${ }^{5}$ Aus der Gebietshoheit folgt damit die Verantwortung, Menschen auf dem eigenen Staatsgebiet vor Menschenrechtsverletzungen zu schützen. Zwar wurde das Konzept

${ }^{2}$ Georg Jellinek, Allgemeine Staatslehre, Bad Homburg ${ }^{3} 1966$, S. 183.

${ }^{3}$ Dazu Christian Tomuschat, Human Rights. Between Idealism and Realism, Oxford ${ }^{3} 2014$, S. $27 \mathrm{ff}$.

${ }^{4}$ Dazu grundlegend: EGMR, Urteil Nr. 8978/80 - X und Y gegen Niederlande, 26.3. 1985, Rn. 23 ff., deutsche Übersetzung in: NJW 38 (1985), S. 2075; alle EGMREntscheidungen sind im englischen und/oder französischen Original abrufbar unter: http://hudoc.echr.coe.int/.

${ }^{5}$ Hierzu und zum Folgenden: International Commission on Intervention and State Sovereignty, The Responsibility to Protect, Ottawa 2001, Rn. 2.14 f. und Rn. 2.16 ff. 
der Schutzverantwortung in späteren UN-Texten auf den notwendigen Schutz vor Völkermord, Kriegsverbrechen, ethnischen Säuberungen und Verbrechen gegen die Menschlichkeit eingeengt. ${ }^{6}$ Der Grundgedanke reicht aber weiter und erstreckt sich ganz allgemein auf die Verantwortung des Staats für gute Lebensbedingungen im eigenen Staatsgebiet.

Die Staatsgewalt erstreckt sich als sogenannte Gebietshoheit auf das eigene Staatsgebiet und als sogenannte Personalhoheit auf die Staatsangehörigen, die das eigene Staatsvolk bilden. Während aber die Personalhoheit des Staats dort, wo sich eigene Staatsangehörige im Ausland befinden, auf natürliche und rechtliche Grenzen stößt, ist die Gebietshoheit über das eigene Staatsgebiet grundsätzlich umfassend und ausschließlich. Sie erstreckt sich auf alle Personen, die sich auf dem Gebiet befinden, gleich ob es sich um eigene Staatsangehörige handelt oder um Fremde, während es Drittstaaten grundsätzlich verwehrt ist, auf dem eigenen Staatsgebiet Hoheitsakte zu setzen. Der Europäische Gerichtshof für Menschenrechte (EGMR) hat dies 2001 in der Banković-Entscheidung auf den Punkt gebracht ${ }^{7}$ : Aus völkerrechtlicher Sicht seien die Hoheitsbefugnisse eines Staats vorrangig territorialer Natur. Auch wenn das Völkerrecht die extraterritoriale Ausübung von Hoheitsgewalt durch einen Staat nicht ausschließe, so würden die möglichen Grundlagen einer solcher Hoheitsgewalt grundsätzlich durch die souveränen gebietsbezogenen Rechte der betroffenen Drittstaaten definiert und begrenzt.

Versteht man Souveränität im Sinne des klassischen Völkerrechts als die ausschließliche Herrschaftsgewalt über das eigene Staatsgebiet,

${ }^{6}$ UN-Generalversammlung, World Summit Outcome (A/RES/60/1), 16. 9. 2005, Rn. 138; http://undocs.org/A/RES/60/1; Implementing the responsibility to protect. Report of the Secretary-General (A/63/677), 12.1.2009, Rn. 11; http:// undocs.org/A/63/677.

${ }^{7}$ EGMR (Große Kammer), Entscheidung Nr. 52207/99 - Banković u. a. gegen Belgien u. a., 12.12.2001, Rn. 59: „from the standpoint of public international law, the jurisdictional competence of a State is primarily territorial. While international law does not exclude a State's exercise of jurisdiction extra-territorially, the suggested bases of such jurisdiction [...] are, as a general rule, defined and limited by the sovereign territorial rights of the other relevant States. "Deutsche Übersetzung in: NJW 56 (2003), S. 413. Zum territorialen Charakter staatlicher Herrschaft aus verfassungstheoretischer Sicht vgl. Klaus Ferdinand Gärditz, Territoriality, Democracy, and Borders: A Retrospective on the „Refugee Crisis“, in: German Law Journal 17 (2016), S.907-922, hier S. 908 ff. 
folgt daraus auch das Recht, den Zugang zum eigenen Staatsgebiet zu kontrollieren. Entsprechende Kontrollen haben sich an der Wende vom 19. zum 20. Jahrhundert zunehmend durchgesetzt, so dass Staaten seit dem Ersten Weltkrieg flächendeckend die Einreise in ihr Hoheitsgebiet überwachen. ${ }^{8}$ Wenn die Europäische Union (EU) nun in ihrem Innern Unionsbürgern gemäß Art. 21 des Vertrags über die Arbeitsweise der Europäischen Union Freizügigkeit gewährt, erscheint dies als regionale Ausnahme von der völkerrechtlichen Regel. Internationale Menschenrechte umfassen zwar die Ausreisefreiheit und das Recht, in seinen eigenen Staat einzureisen. ${ }^{9}$ Es gibt aber kein Recht, in einen fremden Staat einzureisen. Der Europäische Gerichtshof für Menschenrechte hat auch dies auf den Punkt gebracht: In ständiger Rechtsprechung betont er, dass das Recht der Vertragsstaaten im Völkerrecht allgemein anerkannt sei, die öffentliche Ordnung vorbehaltlich ihrer vertraglichen Verpflichtungen insbesondere auch dadurch aufrechtzuerhalten, dass sie die Einreise und den Aufenthalt von Ausländern kontrollieren. ${ }^{10}$

\section{Das territoriale Asyl}

Das Institut des territorialen Asyls baut gleich in doppelter Weise auf dem Konzept souveräner Staatlichkeit auf. ${ }^{11}$ Zum einen handelt es sich um das Recht des souveränen Staats, Flüchtlingen auf seinem eigenen Staatsgebiet Schutz zu gewähren. Zum anderen knüpft die Schutzgewähr im klassischen Flüchtlingsrecht daran an, dass sich der Heimatstaat, der an sich zum Schutz verpflichtet wäre, gegen seinen Staatsangehörigen wendet und diesen politisch verfolgt. Damit basiert das

${ }^{8}$ Vgl. Vincent Chetail, The transnational movement of persons under general international law - Mapping the customary law foundations of international migration law, in: ders./Céline Bauloz (Hrsg.), Research Handbook on International Law and Migration, Cheltenham/Northampton 2014, S.1-72, hier S.29-32. ${ }^{9}$ Exemplarisch Art. 12 Abs. 2 und 4 des Internationalen Pakts über bürgerliche und politische Rechte vom 19.12.1966, BGBl. 1973 II, S.1534. Vgl. auch Art. 2 Abs. 2, Art. 3 Abs. 2 des 4.Zusatzprotokolls zur Europäischen Menschenrechtskonvention, BGBl. 2002 II, S. 1074.

${ }^{10}$ Vgl. EGMR, Urteil Nr. 26102/95 - Dalia gegen Frankreich, 19.2.1989, Rn. 52; EGMR (Große Kammer), Urteil Nr. 48321/99 - Slivenko u. a. gegen Lettland, 9.10.2003, Rn. 115; ähnlich EGMR (Große Kammer), Urteil Nr.37201/06 - Saadi gegen Italien, 28.2.2008, Rn. 124.

${ }^{11}$ Zum territorialen Charakter von Asyl und Flüchtlingsschutz vgl. auch Gärditz, Territoriality, S. $913 \mathrm{f}$. 
Konzept der politischen Verfolgung auf einem Schutzentzug durch den Heimatstaat, wie er nun auch in der Theorie der staatlichen Schutzverantwortung thematisiert wird.

Kraft ihrer Gebietshoheit sind Staaten berechtigt, Fremden auf ihrem eigenen Staatsgebiet Schutz vor Verfolgung zu gewähren. Asyl ist damit ein staatliches Recht oder eine staatliche Befugnis, aber kein Individualrecht, das einzelnen Flüchtlingen zustehen würde. Art. 14 der Allgemeinen Erklärung der Menschenrechte, die am 10. Dezember 1948 von der Generalversammlung der Vereinten Nationen verabschiedet wurde, postuliert zwar ein Recht, Asyl zu suchen und zu genießen. ${ }^{12}$ Ein Recht, Asyl zu erhalten, wurde aber gerade nicht festgeschrieben. ${ }^{13}$ Auch die Genfer Flüchtlingskonvention (GFK) vom 28. Juli $1951^{14}$ gewährt kein solches Recht. Als „Magna Charta des Flüchtlingsrechts“ definiert sie zwar den völkerrechtlichen Flüchtlingsbegriff und normiert Schutzstandards für Flüchtlinge, die in einem Vertragsstaat Aufnahme gefunden haben. Das Recht auf Aufnahme bleibt aber aus geklammert. Vielmehr begnügt sich die Konvention insoweit mit einem Mindestschutz in Form des Non-Refoulement gemäß Art. 33. Ein Individualrecht auf Asyl ist nationalen Verfassungsbestimmungen wie Art. 16a Abs. 1 des deutschen Grundgesetzes vorbehalten. ${ }^{15}$ Selbst Art. 18 der Europäischen Grundrechtecharta bleibt zumindest mehrdeutig. Dort ist zwar von einem „Recht auf Asyl“ die Rede; dieses soll aber nur nach Maßgabe der Genfer Flüchtlingskonvention garantiert werden, die gerade kein Recht auf Aufnahme gewährt.

Der völkerrechtliche Status quo wurde von den Staaten im Herbst 2016 auf dem Flüchtlings- und Migrationsgipfel zum Auftakt der UN-Generalversammlung bekräftigt: „We reaffirm respect for the institution of asylum and the right to seek asylum." ${ }^{16}$ Asyl ist danach ein objektiv-rechtliches Rechtsinstitut. Anerkannt wird zwar das Individualrecht, Asyl zu suchen, nicht aber das Recht, Asyl zu erhalten. Die Wort-

${ }^{12}$ Im Original: „Everyone has the right to seek and to enjoy in other countries asylum from persecution“; A/RES/217A(III), http://undocs.org/A/RES/217(III). ${ }^{13} \mathrm{Vgl}$. dazu anschaulich Otto Kimminich, Die Entwicklung des internationalen Flüchtlingsrechts - faktischer und rechtsdogmatischer Rahmen, in: AVR 20 (1982), S. 369-410, hier S.403 f.

${ }^{14}$ Abgedruckt in BGBl. 1953 II, S.560-583.

${ }^{15}$ Vgl. dazu den Beitrag von Agnes Bresselau von Bressensdorf in diesem Band. ${ }^{16}$ New York Declaration for Refugees and Migrants (A/RES/71/1), 19.9. 2016, Rn. 67; http://undocs.org/A/RES/71/1. 
wahl macht deutlich, dass die Staaten auch 2016 nicht bereit waren, über das Versprechen von 1948 hinauszugehen.

Blickt man auf den Herkunftsstaat, knüpft das politische Asyl mit dem Konzept der politischen Verfolgung an die Schutzverantwortung des Herkunftstaats an. ${ }^{17}$ Nach dem oben zur Schutzverantwortung Gesagten, ist es Aufgabe eines jedes Staats, auf seinem Staatsgebiet ein menschenwürdiges Leben zu gewährleisten. Wer im Ausland bedroht wird, kann in seinen Heimatstaat zurückkehren, und der Heimatstaat kann sich Kraft seiner Personalhoheit auf dem Weg des konsularischen und diplomatischen Schutzes für die Belange seiner Staatsangehörigen einsetzen. Wer aber von seinem eigenen Heimatstaat im Stich gelassen wird, steht schutzlos da. Auf diese Situation besonderer Schutzbedürftigkeit dürfen andere Staaten mit der Gewährung politischen Asyls reagieren

Dieser Zusammenhang von Schutzdefizit und externem Schutz findet sich bereits in den Anfängen des Flüchtlingsvölkerrechts. Im Zuge der russischen Revolution flohen viele Vertreter des zaristischen Russland aus der neu entstandenen Sowjetunion. Das galt insbesondere für die Weißen Garden, nachdem sie der Roten Armee unterlegen waren. Allein in Konstantinopel befanden sich Anfang der 1920er Jahre unter widrigsten Bedingungen 25.000 Flüchtlinge der ehemaligen Armee des russischen Generals Peter Wrangel..$^{18}$ Insgesamt waren bald 900.000 Menschen in die Nachbarstaaten des einstigen russischen Imperiums geflohen. In dieser dramatischen Situation berief der Völkerbund im September 1921 den Antarktisforscher Fridtjof Nansen zum Flüchtlingshochkommissar. Nansen erhielt vom Völkerbund das vorübergehende Mandat zur Unterstützung ,jeder Person russischen Ursprungs, die den Schutz der Regierung der UdSSR nicht oder nicht mehr genießt und die keine andere Staatsangehörigkeit erworben hat“ “. ${ }^{19}$ Es ging also um Personen, die als russische Staatsangehörige der Personalhoheit der UdSSR unterstanden oder - im Falle der Ausbürgerung - unterstanden hatten. Diese Personen zu schützen war zunächst Aufgabe der UdSSR. Da ihnen der Heimatstaat jedoch den Schutz entzogen hatte, waren sie schutzlos, so dass es nun der interna-

\footnotetext{
${ }^{17}$ Vgl. Hugo Storey, Persecution: Towards a working definition, in: Chetail/Bauloz (Hrsg.), Research Handbook, S. 459-518, hier S.480-483.

${ }^{18}$ Vgl. Claudena M. Skran, Refugees in Inter-War Europe. The Emergence of a Regime, Oxford 1995, S.185-189; die folgende Angabe findet sich ebd., S. 35.

${ }^{19}$ Zit. nach Peter Gatrell, The Making of the Modern Refugee, Oxford 2015, S. 55.
} 
tionalen Gemeinschaft oblag, diese Personen zu schützen. Der Schutz endet damals wie heute in dem Augenblick, in dem ein Flüchtling die Staatsangehörigkeit eines anderen Staats erwirbt, weil er dann wieder unter dem Schutz eines Staats steht, dem er angehört.

Nach dem Zweiten Weltkrieg wurde der Flüchtlingsbegriff, der sich in der Zwischenkriegszeit herausgebildet hatte, in der Genfer Flüchtlingskonvention aufgegriffen. Gemäß Art. 1 A Nr. 2 GFK ist Flüchtling jede Person, die

„aus der begründeten Furcht vor Verfolgung wegen ihrer Rasse, Religion, Nationalität, Zugehörigkeit $\mathrm{zu}$ einer bestimmten sozialen Gruppe oder wegen ihrer politischen Überzeugung sich außerhalb des Landes befindet, dessen Staatsangehörigkeit sie besitzt, und den Schutz dieses Landes nicht in Anspruch nehmen kann oder wegen dieser Befürchtung nicht in Anspruch nehmen will“.

Erforderlich sind also sowohl eine Diskriminierung, die den Einzelnen aus dem Gros der Bevölkerung aussondert, als auch ein gewisser Verfolgungsdruck. Mit den aufgelisteten Merkmalen waren Oppositionelle, die die Sowjetunion wegen ihrer politischen Überzeugungen verlassen mussten, ebenso erfasst wie beispielsweise Menschen, die vom NS-Regime allein wegen ihrer jüdischen Herkunft verfolgt wurden. Der enge Flüchtlingsbegriff des geltenden Völkerrechts ist demnach vor dem Hintergrund der Verfolgungsschicksale der ersten Hälfte des 20. Jahrhunderts zu verstehen. Die Verfolgungen, die namentlich von der Sowjetunion und vom NS-Regime ausgingen, führten zu einer Welle an Hilfsbedürftigen. Gleichzeitig herrschte in den westlichen Staaten, die sich vom Kommunismus ebenso abzugrenzen suchten wie vom Faschismus, ein relativ breiter Konsens, dass den Betroffenen internationale Hilfe zuteilwerden musste. Dementsprechend waren die Hilfsmaßnahmen des Völkerbunds und später die Genfer Flüchtlingskonvention genau auf diese Gruppen zugeschnitten; auch Art. 16a des Grundgesetzes orientiert sich an diesem Konzept.

Bezeichnenderweise war die Genfer Flüchtlingskonvention in ihrem Art.1 A Nr. 2 zunächst auf Fluchtgründe begrenzt, die vor dem 1. Januar 1951 entstanden waren. Erst Art. I Abs. 2 des Protokolls vom 31. Januar $1967^{20}$ hat diese Begrenzung aufgehoben und die Genfer Flüchtlingskonvention zu einem allgemeinen Instrument für zukünftige Ereignisse gemacht. Die Frontstellung gegen den Kommunismus wirkte jedoch

${ }^{20}$ Abgedruckt in BGBl. 1969 II, S. 1298. 
noch während des Kalten Kriegs nach. Das gilt sowohl für den Umgang mit vietnamesischen Bootsflüchtlingen seit den 1970er Jahren als auch für die afrikabezogene Flüchtlingshilfe in den 1980er Jahren. ${ }^{21}$ Mit ihrem engen Flüchtlingsbegriff lässt die Genfer Flüchtlingskonvention große Gruppen von Personen, die mehr oder minder unter Druck ihre Heimat verlassen, ungeschützt. Das gilt zunächst für Kriegs- und Bürgerkriegsflüchtlinge, die vor den tödlichen Gefahren massiver kriegerischer Gewalt fliehen. Werden sie im Heimatstaat gerade wegen ihrer Religionszugehörigkeit oder als Angehörige der politischen Opposition angegriffen, wie es etwa in Syrien teilweise der Fall ist, so fallen sie unter den Flüchtlingsbegriff der Genfer Flüchtlingskonvention. Finden sie sich aber schlicht zwischen den Fronten oder sonst unter feindlichem Beschuss wieder, ohne dass die Angriffe durch ihre spezifische Gruppenzugehörigkeit motiviert wären, so fallen sie als reine Gewaltflüchtlinge nicht in den Anwendungsbereich der Genfer Flüchtlingskonvention. ${ }^{22}$ Freilich geht das Flüchtlingshochkommissariat der Vereinten Nationen, das über die Anwendung der Genfer Flüchtlingskonvention wacht, in solchen Fällen pragmatisch vor und stellt dort, wo es mit Gruppen von Gewaltflüchtlingen konfrontiert ist, deren Schutzbedürftigkeit auf einer prima-facie-Basis fest. ${ }^{23}$

Noch klarer ist der Ausschluss bei denen, die lediglich vor wirtschaftlicher Not fliehen. Zwar kann auch wirtschaftliche Not das Ergebnis politischer Verfolgung sein, wenn etwa ein Heimatstaat bestimmte Bevölkerungsgruppen systematisch hungern und verhungern lässt. Ist jedoch die Wirtschaftslage allgemein desolat, stellt auch existenzielle wirtschaftliche Not keine politische Verfolgung im Sinne der Genfer Konvention dar. Verlassen die Betroffenen getreu dem Motto der Bremer Stadtmusikanten, „etwas Besseres als den Tod findest du überall“, ihre Heimat, sind sie in gängigen Kategorien bloße „Wirtschaftsflüchtlinge“, denen wenig Verständnis entgegengebracht wird.

${ }^{21}$ Vgl. Penelope Mathew/Tristan Harley, Refugees, Regionalism and Responsibility, Cheltenham 2016, S.161, 165 und 172.

${ }^{22}$ Vgl. Andreas Zimmermann/Claudia Mahler, Art. 1 A, para. 21951 Convention, in: Andreas Zimmermann (Hrsg.), The 1951 Convention Relating to the Status of Refugees and its 1967 Protocol. A Commentary, Oxford 2011, S.281-466, hier Rn. 315 ff.

${ }^{23}$ Dazu T. Alexander Aleinikoff, The mandate of the Office of the United Nations High Commissioner for Refugees, in: Chetail/Bauloz (Hrsg.), Research Handbook, S.389-415, hier S.396 f. 
Die traditionelle Privilegierung politisch Verfolgter mag noch immer ihre Berechtigung haben, weil sich diejenigen, die von ihrem Heimatstaat gezielt verfolgt werden, tatsächlich in einer besonders dramatischen Lage befinden. Kann ein Staat aber seiner Schutzverpflichtung angesichts eines bewaffneten Konflikts oder einer existenziellen Wirtschaftskrise insgesamt nicht mehr nachkommen, ist dies für die Einzelnen nicht minder lebensbedrohend. Das Schutzbedürfnis dürfte ähnlich sein, nur dass die Empathie der Staatengemeinschaft deutlich geringer ausfällt. Lässt man die hehren Ideen des Flüchtlingsrechts der Zwischen- und Nachkriegszeit hinter sich, fällt die völkerrechtliche Bilanz des territorialen Asyls mager aus. Ein Individualrecht gibt es nicht, und es werden auch nur relativ wenige Betroffene erreicht. Das Gros der Gewalt- und „Wirtschaftsflüchtlinge“ hat keine Chance auf Asyl.

\section{Der völkerrechtliche Mindestschutz: Non-Refoulement}

Umso wichtiger wird der völkerrechtliche Mindestschutz gegen Abund Rückschiebung. Dieses Gebot des Non-refoulement findet sich in Art. 33 Abs. 1 GFK:

„Keiner der vertragsschließenden Staaten wird einen Flüchtling auf irgendeine Weise über die Grenzen von Gebieten ausweisen oder zurückweisen, in denen sein Leben oder seine Freiheit wegen seiner Rasse, Religion, Staatsangehörigkeit, seiner Zugehörigkeit zu einer bestimmten sozialen Gruppe oder wegen seiner politischen Überzeugung bedroht sein würde.“

Wie die Flüchtlingsdefinition knüpft auch das Verbot des Refoulement an eine drohende Verfolgung wegen eines diskriminierenden Merkmals an, wobei der Verfolgungsdruck mit der Gefahr für Leben oder Freiheit besonders hoch sein muss. Das Refoulement-Verbot setzt also eine qualifizierte Form der politischen Verfolgung voraus. Während im Normalfall die Aufnahme eines politisch Verfolgten im freien Ermessen des anderen Staats steht, besteht hier die Verpflichtung, Personen, die den sicheren Staat erreicht haben, nicht in einen Staat zurückzuweisen, in dem sich die Verfolgung aktualisiert.

Freilich ist nur die Abschiebung in den Verfolgerstaat unzulässig. Art. 33 Abs. 1 GFK hindert einen Staat nicht daran, einen Flüchtling in einen Drittstaat zurückzuschieben, solange nur die Gewähr besteht, dass er dort den Mindestschutz erhält und nicht im Wege der sogenannten Kettenabschiebung von dort direkt oder indirekt in einen 
anderen Staat abgeschoben wird, in dem sich die Verfolgung aktualisiert. ${ }^{24}$ Betrachtet man allein die GFK und lässt Unionsrecht außer Betracht $^{25}$, dürfte Deutschland also jeden Flüchtling, der aus Österreich nach Bayern gelangt, nach Österreich zurückschieben, solange Österreich seine Verpflichtungen aus Art. 33 Abs. 1 GFK wahrt.

Seit Ende der 1980er Jahre hat der Europäische Gerichtshof für Menschenrechte auch aus der Europäischen Menschenrechtskonvention (EMRK) ${ }^{26}$ ein Refoulement-Verbot abgeleitet. Grundlage ist Art. 3 EMRK, der Folter sowie jede unmenschliche oder erniedrigende Behandlung und Bestrafung verbietet. Das konventionsrechtliche Refoulement-Verbot beruht auf dem Grundgedanken, es sei unmenschlich, einen Menschen in einen Staat ab- oder zurückzuschieben, in dem ihm Folter, unmenschliche oder erniedrigende Behandlung drohe. Nach der Leitentscheidung in der Sache Cruz Varas ${ }^{27}$ ist eine Abschiebung dann unzulässig, wenn „wesentliche Gründe“ für die Annahme vorliegen, dass für den Fall der Rückkehr in das andere Land die „tatsächliche Gefahr“ einer unmenschlichen oder erniedrigenden Behandlung bestehe. Dieser Schutz besteht nicht nur dann, wenn sich ein Flüchtling bereits einige Zeit auf dem eigenen Staatsgebiet aufgehalten hat. Vielmehr verbietet es Art. 3 EMRK auch, einen Menschen an der Grenze abzuweisen, wenn ihm im anderen Staat eine Behandlung droht, die Art. 3 EMRK widerspricht, oder wenn er Gefahr läuft, im Wege einer „Kettenabschiebung“ in einen anderen Staat weitergeschoben zu werden, in denen die Standards des Artikels 3 EMRK nicht gewahrt sind. Auf dieser Grundlage hat der EGMR bereits 2011 in der Sache M.S.S. gegen Belgien und Griechenland die völlige Unzulänglichkeit des Flüchtlingsschutzes in Griechenland festgestellt - mit der Folge, dass in der Europäischen Union (EU) kein Flüchtling, der über Griechenland eingereist war, mehr dorthin rücküberstellt werden durfte. ${ }^{28}$ Damit musste jedem deutschen Innenminister spätestens vier

${ }^{24}$ Vgl. Walter Kälin/Martina Caroni/Lukas Heim, Art. 33, para. 11951 Convention, in: Zimmermann (Hrsg.), Convention, S.1327-1396, hier Rn. 145 ff.

${ }^{25} \mathrm{Zu}$ diesbezüglichen Schranken vgl. Anuscheh Farahat/Nora Markard, Forced Migration Governance. In Search of Sovereignty, in: German Law Journal 17 (2016), S. 923-947, hier S.930-933.

${ }^{26}$ Abgedruckt in BGBl. 2002 II, S.1055-1123.

${ }^{27}$ Dazu EGMR, Urteil Nr. 15576/89 - Cruz Varas gegen Schweden, 20. 3.1991, Rn. 69 f., deutsche Übersetzung in: NJW 44 (1991), S. 3079.

${ }^{28}$ Dazu EGMR (Große Kammer), Urteil Nr.30696/09 - M.S.S. gegen Belgien u. a., 21.1.2011, Rn. 294 ff., deutsche Übersetzung in: NVwZ 30 (2011), S. 413. 
Jahre, bevor im Sommer 2015 ungezählte Flüchtlinge die bayerische Grenze erreichten, klar sein, dass das Asylsystem der EU nicht funktionierte. ${ }^{29}$

Das konventionsrechtliche Refoulement-Verbot ist insofern weiter als Art. 33 Abs. 1 GFK, als es jede unmenschliche oder erniedrigende Behandlung erfasst. Die Diskriminierung wegen einer bestimmten Gruppenzugehörigkeit, die den Begriff der politischen Verfolgung ausmacht, ist bei Art. 3 EMRK nicht erforderlich, so dass beispielsweise auch bloße Gewaltflüchtlinge geschützt sein können. Außerdem eröffnet Art. 3 EMRK mit der Beschwerde zum EGMR den Zugang zu einem hoch entwickelten internationalen Kontrollsystem.

Es bleibt die Frage, wann ein Flüchtling das Staatsgebiet dergestalt erreicht hat, dass eine Rückschiebung nur noch nach Maßgabe von Art. 33 Abs. 1 GFK möglich ist. Sicher ist das der Fall, wenn die Person bereits über die Grenze gelangt ist und deutschen Boden betreten hat. Dabei bestimmt Art. 31 Abs. 1 GFK zusätzlich, dass die irreguläre Einreise in Fällen, in denen Art. 33 Abs. 1 GFK eingreift, nicht kriminalisiert und als Straftat verfolgt werden darf. Auch dann, wenn die Person auf einem deutschen Flughafen landet, hat sie deutschen Boden betreten, und zwar unabhängig davon, ob sie die Grenzkontrolle durchschritten hat oder nicht. Finden die Grenzkontrollen erst auf deutschem Boden statt, befindet sich die Einreise begehrende Person bereits auf dem Gebiet, auf dem der Staat die Menschen- und Grundrechte garantieren muss. Verfahren für das Flughafenasyl müssen also in vollem Umfang Art. 33 GFK sowie Art. 3 EMRK wahren. Nichts anderes gilt, wenn ein Flüchtling von der Bundespolizei im Passauer Bahnhof im IntercityExpress aufgegriffen wird.

Darüber hinaus wird das Refoulement-Verbot so weit verstanden, dass es schon dann greift, wenn ein Flüchtling an der Landgrenze Einlass begehrt. ${ }^{30}$ Kann nicht ausgeschlossen werden, dass einer Person bei der Abweisung Verfolgung im Sinne von Art. 33 GFK droht, muss

${ }^{29}$ Vgl. auch Robert Uerpmann-Wittzack, Die Europäische Menschenrechtskonvention: Gemeinsamer Mindeststandard oder Vollharmonisierung des Grundrechtsschutzes in Europa?, in: Jens Peter Brune/Heinrich Lang/Micha H. Werner (Hrsg.), Konzepte normativer Minimalstandards. Ethische und rechtliche Perspektiven, Baden-Baden 2016, S. 143-175, hier S.150-154.

${ }^{30}$ Vgl. Kälin/Caroni/Heim, Art. 33, para. 1, in: Zimmermann (Hrsg.), Convention, Rn. 105 ff.; Roberta Mungianu, Frontex and Non-Refoulement. The International Responsibility of the EU, New York 2016, S. 140 ff. 
ihr also zumindest vorübergehend die Einreise gestattet werden. In der Sache Hirsi Jamaa ist der EGMR noch weiter gegangen und hat das menschenrechtliche Rückschiebungsverbot auch auf Fälle ausgedehnt, in denen europäische Küstenwach- oder Kriegsschiffe Flüchtlinge auf hoher See aufgreifen. ${ }^{31}$ Nehmen sie Flüchtlinge an Bord oder übernehmen sie die Kontrolle über das fremde Boot, so unterstellen sie die Flüchtlinge der eigenen Hoheitsgewalt mit der Folge, dass gemäß Art. 1 EMRK deren Garantien greifen. Art. 3 EMRK verbietet es der Besatzung des Schiffs dann, die aufgegriffenen Personen in einen Drittstaat zu bringen, wenn ihnen dort oder durch eine zu befürchtende Weiterschiebung eine unmenschliche oder erniedrigende Behandlung droht. Streckt ein europäischer Staat also mit seiner Küstenwache oder Marine den kleinen Finger aus und rettet Flüchtlinge aus Seenot, so können diese die ganze Hand ergreifen und über Art. 3 EMRK de facto vielfach die Einreise in ein europäisches Land erzwingen. Das Dilemma lässt sich de lege lata kaum lösen. Gleichzeitig bedeutet das, dass Kontrollen auf hoher See, die die Einreise von Flüchtlingen verhindern sollen, kaum Wirkung zeigen können. Die Alternative, sich aus dem Mittelmeer weitgehend zurückzuziehen und dieses zum Grab für unzählige Flüchtlinge werden zu lassen, widerspräche nicht nur den Vereinbarungen zur Seenotrettung ${ }^{32}$, sondern erscheint auch moralisch inakzeptabel.

Angesichts des weiten Anwendungsbereichs des Non-RefoulementPrinzips haben die europäischen Staaten verschiedene Ausweichstrategien entwickelt. Der Grundgedanke ist das Outsourcen der Grenzkontrolle auf Dritte. Eine Strategie besteht darin, Fluggesellschaften haftbar zu machen, wenn sie Personen ohne gültige Einreisepapiere ins Inland bringen. So verbietet es $\S 63$ Abs. 1 AufenthG Fluggesellschaften, einen Ausländer ins Inland zu bringen, der die notwendigen Einreisepapiere nicht besitzt. Gemäß $\S 63$ Abs. 2 AufenthG kann die zuständige Behörde das Verbot im Einzelfall konkretisieren und dann mit einem Zwangsgeld durchsetzen. § 64 AufenthG verpflichtet das Luftfahrtunternehmen, zurückgewiesene Ausländer zurückzubefördern. Damit wird die Einreisekontrolle den Fluggesellschaften übertragen, die sie

${ }^{31}$ So EGMR (Große Kammer), Urteil Nr.27765/09 - Hirsi Jamaa u. a. gegen Italien, 23.2.2012, Rn. 123-138, deutsche Übersetzung in: NVwZ 31 (2012), S. 809.

${ }^{32}$ Vgl. dazu Nora Markard, Das Recht auf Ausreise zur See. Rechtliche Grenzen der europäischen Migrationskontrolle durch Drittstaaten, in: AVR 52 (2014), S. 449-494, hier S. 477 ff. 
bereits vor dem Abflug durchführen. Selbst wenn der Staat den Transport politisch Verfolgter nicht sanktioniert, entfaltet die Regelung ihre volle Wirkung, weil Fluggesellschaften kaum das Haftungsrisiko für den Fall eingehen werden, dass eine Person nicht als Flüchtling anerkannt wird. Der Rückschiebeschutz der Art. 33 Abs. 1 GFK und Art. 3 EMRK wird also effektiv ausgehebelt, indem die Kontrolle auf private Akteure und ins Ausland verlagert wird. Ähnlich verhält es sich mit Abkommen zwischen Deutschland, anderen EU-Mitgliedstaaten oder der EU mit anderen Mittelmeeranrainern, in denen sich letztere verpflichten, potenzielle Flüchtlinge nicht ausreisen zu lassen. Auch hier wird die Grenzkontrolle ausgelagert, so dass Flüchtlinge nicht mehr in den räumlichen Anwendungsbereich der Art. 33 Abs. 1 GFK und Art. 3 EMRK gelangen. Allerdings kollidieren derartige Vereinbarungen noch deutlicher als die Inpflichtnahme von Fluggesellschaften mit der völkerrechtlich verbürgten Ausreisefreiheit. ${ }^{33}$ Während die Ausreisefreiheit im Westeuropa des Kalten Krieges als Antwort auf den Eisernen Vorhang hochgehalten wurde ${ }^{34}$, tritt sie heute im rechtlichen Bewusstsein zurück.

\section{Zusammenfassung}

Das gegenwärtige Völkerrecht wird also gerade auch im Flüchtlingsrecht durch das Konzept territorial definierter staatlicher Herrschaft geprägt. Als territorialer Souverän ist jeder Staat berechtigt, den Zugang zum eigenen Staatsgebiet zu kontrollieren und Fremde gegebenenfalls auszuschließen. Allerdings ist territoriale Herrschaft mit Verantwortung verbunden. Kehrt sich ein Staat gegen den eigenen Staatsangehörigen, den er eigentlich zu schützen hätte, so dass dieser fliehen muss, bedarf der Flüchtling internationalen Schutzes. Allerdings bleibt die Aufnahme von Flüchtlingen eine souveräne Entscheidung des einzelnen Staats. Es gibt also kein völkerrechtliches Asylrecht, das das souveräne Recht zur Grenzkontrolle einschränken würde. Erst im Refoulement-Verbot findet das souveräne Recht der Grenzkontrolle seine Grenzen. So betont der EGMR, dass das völkerrechtlich allgemein

${ }^{33}$ Vgl. ebd., S. 456 ff.

${ }^{34}$ Vgl. etwa Rainer Hofmann, Die Ausreisefreiheit nach Völkerrecht und staatlichem Recht, Berlin 1988, S. 9 ff., und Robert Uerpmann, Die Ausreise von DDRBürgern aus Ungarn aus völkerrechtlicher Sicht, in: Juristische Ausbildung 12 (1990), S. 12-16, hier S 14 f. 
anerkannte Recht des einzelnen Staats, Einreise und Aufenthalt von Fremden zu kontrollieren, nur durch die vertraglich eingegangenen Verpflichtungen des Staats begrenzt würden. Art. 33 GFK und Art. 3 EMRK sind derartige Verpflichtungen, die die Freiheit der Staaten im Individualinteresse beschränken und den Staat dazu verpflichten, bestimmten Personen zumindest subsidiären Schutz zu gewähren. 